\title{
Editorial: Topical Collection on Understanding the Diversity of Planetary Atmospheres
}

\author{
François Forget ${ }^{1}$ - Oleg Korablev ${ }^{2}$. Julia Venturini ${ }^{3}$. \\ Takeshi Imamura ${ }^{4}$ Helmut Lammer ${ }^{5}$ Michel Blanc ${ }^{6}$
}

Received: 10 March 2021 / Accepted: 10 March 2021 / Published online: 19 April 2021

(C) The Author(s), under exclusive licence to Springer Nature B.V. 2021

Ten planetary atmospheres are currently studied in the solar system and many more will be characterized in the coming years as we remotely observe exoplanets. Are we ready to understand what we will discover around other stars? The examples of the solar system are probably not sufficient to let us anticipate the diversity of exo-atmospheres. To prepare this revolution, it is nevertheless very interesting to make the most of what we have learned so far, to identify commonalities between the different solar system atmospheres, and to make out the remaining key questions in our understanding of the known planetary atmospheres.

Toward these goals, we propose a series of articles gathered within a Space Science Reviews Topical Collection entitled "Understanding the Diversity of Planetary Atmospheres". It was prepared during a workshop organized at the International Space Science Institute in Bern, on November 12-16, 2018, with the support of the Europlanet Research Infrastructure of the EU. Nearly 40 scientists from Europe, USA, Russia, China, Japan, Israel and Australia attended this meeting, including planetary scientists, experts in the origins of atmospheres, planetary interior, aeronomy, escape, climatologists, and astronomers. The diversity of expertise proved to be very fruitful to discuss the diversity of atmospheres.

Understanding the Diversity of Planetary Atmospheres

Edited by François Forget, Oleg Korablev, Julia Venturini, Takeshi Imamura, Helmut Lammer and Michel Blanc

F. Forget

1 Laboratoire de Météorologie Dynamique/IPSL, Sorbonne Université, ENS, PSL Research University, Ecole Polytechnique, CNRS, Paris, France

2 Space Research Institute (IKI), Russian Academy of Sciences, 84/32 Profsoyuznaya, 117997, Moscow, Russia

3 International Space Science Institute, Hallerstrasse 6, 3012, Bern, Switzerland

4 Graduate School of Frontier Sciences, The University of Tokyo, Kashiwa, Japan

5 Space Research Institute, Austrian Academy of Sciences, Graz, Austria

6 IRAP, CNRS-UPS-CNES, Toulouse, France 
A first, extremely difficult question is the anticipation of the possible atmospheric compositions and masses that control the environment on any world. These depend on complex processes which remain difficult to model: the origins of volatiles, atmospheric escape, geochemistry, photochemistry, etc. Initially, the volatiles that will compose the atmosphere originate from the different building blocks present in protoplanetary discs and from the different physical and chemical processes that they experience during the planetary assembly and evolution. To set the stage, J. Venturini, M.P. Ronco and O.M. Guilera summarise, in a nutshell, the key concepts and processes operating during planet formation, with a focus on the delivery of volatiles to the inner regions of the planetary system that ultimately controls planetary habitability. Once the different elements that can constitute an atmosphere have been delivered (typically C-H-O-N-S), a large part can be sequestered in the core and mantle. A first atmosphere can be outgassed via "magmatic pipelines" (magma ocean early on, and later volcanism), lost to space, replaced by a secondary atmosphere, etc. In their review of these processes, F. Gaillard, A. Bouhifd, E. Furi et al. detail how atmospheric composition is just what is left from what is taken in the core and the mantle, and how this varies depending on the planetary size, its oxidation state, the prevailing temperature and pressure or the styles of mantle convection (i.e. plate tectonics).

For a given volatile inventory, the actual properties of atmospheres are then controlled by the numerous chemical reactions that not only control the gaseous composition, but also the possible formation of hazes and aerosols. F.P. Mills, J.I. Moses, P. Gao and S.-M. Tsai provide an up-to-date, synthetic review on what we have learned from the commonalities in the diverse atmospheric chemistry of the solar system, and how they prepare us for the study of exoplanets. Another key process controlling the evolution of atmospheres is atmospheric escape. Many reviews are already available on the subject, but there is one key burning question that deserved to be addressed regarding the possible loss of atmosphere from terrestrial planets: Do intrinsic magnetic fields protect planetary atmospheres from stellar winds? This question is the title of an article provided by R. Ramstad and S. Barabash: on the basis of the ion flux measurements now available from long-lived spacecraft orbiting the solar system's terrestrial planets, they show that, contrary to the current paradigm, an intrinsic magnetic dipole field may not prevent stellar wind-driven escape of planetary atmospheres, and that the presence of one may instead increase the rate of ion escape. They argue that, to explain the evolution of exoplanetary atmospheres, a "modern, nuanced, and evidence-based view of the magnetic field's role in atmospheric escape is required".

As mentioned above, there are currently only ten atmospheres in the solar system that are thick enough to be global, controlled by the equations of meteorology, and able to carry clouds and aerosols: Venus, Earth, Mars, Jupiter, Saturn, Titan, Uranus, Neptune, Triton and Pluto. However, it is interesting to note that much has been learned not only by studying the present-day atmospheres, but also by trying to understand how they have evolved in the past and in particular what were their conditions 3 to 4.4 billion years ago, when the Sun was a younger, fainter, more active star than today. In this spirit, B. Charnay et al. and $M$. Scherf and H. Lammer provide very interesting reviews on key questions in early solar system climatology: "Is the Faint Young Sun Problem for Earth Solved?", "Did Mars Possess a Dense Atmosphere During the First 400 Million Years?". Addressing these questions requires us to discuss many processes relevant to our investigation on the diversity of planetary atmospheres, and has far-reaching consequences for our thinking on planetary habitability.

The study of the atmospheres in the solar system and recent investigations motivated by exoplanet observations have revealed another source of spectacular diversity within the known planetary atmospheres: atmospheric dynamics. A zoo of possible circulation regimes have been identified and many processes remain enigmatic. Four up-to-date review articles 
are included in the Topical Collection, describing our current knowledge and understanding of the supperotation in planetary atmospheres (T. Imamura et al.), the belt/zone circulation of giant planet atmospheres ( $L$. Fletcher et al.) completed by a review of the deep atmospheric dynamics of Jupiter and Saturn recently revealed by the Juno and Cassini gravity measurements ( . Kaspi et al.). The late Adam Showman also led a review study on the Atmospheric Dynamics of hot giant planets and brown dwarfs (A. Showman, X. Tan and $V$. Parmentier). Shortly after submitting this work, Adam Showman - a major figure in atmospheric and planetary sciences who had contributed to make the most of our workshop on atmospheric diversity- suddenly passed away on 16 March 2020 at the age of 51. This collection is dedicated to him.

The hot giant planets discussed by Adam Showman provide the best observed exoplanetary atmospheres so far. Smaller (terrestrial) planets' atmospheres remain difficult to characterize, but they will soon be observable thanks to a new generation of extremely large terrestrial observatories and space telescopes like the James Webb Space Telescope (JWST). What can we expect? This is the topic of the prospective article proposed by J.L. Grenfell et al. who discuss the possible range of atmospheres on low mass exoplanets, on the basis of available models of planet formation and evolution, building on the lessons from the solar system. Among the possible cases not found in the solar system are low mass planets possessing hydrogen-dominated atmospheres. Yet, more and more "super-Earths" and "mini-Neptunes" are discovered close to their star (with semi-major axes $<1 \mathrm{AU}$ ) and a significant fraction of them have been inferred to possess a voluminous $\mathrm{H} / \mathrm{He}$ atmosphere, including planets with masses as low as 2 Earth masses. J.E. Owen, I.F. Shaikhislamov, $H$. Lammer et al. review the evidence for hydrogen dominated atmospheres on terrestrial planets and discuss the possible origins and evolution of these atmospheres. They suggest that these hydrogen dominated atmospheres must have been accreted from the natal protoplanetary disc, in contradiction with the Solar System terrestrial planets that could only accrete thin hydrogen dominated atmospheres. Within that context, they put forward the role played by hydrodynamic atmospheric escape driven by the stellar high-energy emission that ultimately shapes what is left on this new kind of worlds.

To fully explore the real diversity of exo-atmospheres, more observations will be needed. In that regard, the most promising target in the near term is the TRAPPIST-1 planetary system made of at least seven transiting terrestrial-size, terrestrial-mass planets all receiving a moderate amount of irradiation. A significant observation time of the JWST will soon be dedicated to the observation of this system. Our current knowledge about the properties of these very important planets is reviewed by M. Turbet et al. For this system as well as for all other observation campaigns, one must not be too optimistic and be aware of the fundamental challenges that will limit our capacity to characterize exoplanets. This is reviewed by J.K. Barstow and K. Heng, who also recommend approaches for future improvements.

Meanwhile, we will continue to gain knowledge by exploring the solar system. In particular, much can still be learned from the complex giant planets which remain poorly understood by many aspects. To this aim, M.D. Hofstadter, L.N. Fletcher, A.A. Simon et al. review in details the opportunity offered by the space agencies, and discuss the atmospheric goals to be addressed by currently approved giant-planet missions as well as missions likely to be considered in the next few years, such as a joint NASA/ESA Ice Giant orbiter with atmospheric probe that could explore Uranus or Neptune. These two worlds have only been briefly visited by Voyager 2 during its flybys of these two planets in 1986 and 1989. Yet, the majority of exoplanets discovered so far appear to be Neptune-like (they are found in much larger numbers than gas giants such as Jupiter). Exploration of our local ice giants could allow us to better characterize and understand this abundant category of exoplanets. 
The 15 articles of this topical collection present rich, up-to-date views on many hot scientific questions relating to planetary atmospheres and the climate of exoplanets. By compiling this collection we hope to provide a solid reference for anyone willing to work towards understanding the diversity of planetary atmospheres.

Publisher's Note Springer Nature remains neutral with regard to jurisdictional claims in published maps and institutional affiliations. 